\title{
Efficacy of high-dose rosuvastatin preloading in patients undergoing percutaneous coronary intervention: a meta-analysis of fourteen randomized controlled trials
}

Yilong Pan ${ }^{1}$, Yuan $\operatorname{Tan}^{2}$, Bin $\mathrm{Li}^{1}$ and Xiaodong $\mathrm{Li}^{1 *}$

\begin{abstract}
Background: Numerous studies have evidenced that statins can reduce the incidence of cardiovascular disease. However, the effects of high-dose rosuvastatin (RSV) preloading in patients undergoing percutaneous coronary intervention $(\mathrm{PCl})$ are controversial.

Objective: We attempted to identify and quantify the potential cardioprotective benefits of high-dose RSV preloading on final thrombolysis in myocardial infarction (TIMI) flow grade, major adverse cardiac events (MACE), and peri-procedural myocardial injury (PMI) in patients undergoing $\mathrm{PCl}$.

Methods: Pubmed, EMBASE, Cochrane Central Register of Controlled Trials and ISI Web of Science databases were systematically searched for randomized controlled trials (RCTs) up to June 2015. We assessed the incidence of MACE and PMI in all enrolled patients for subgroups stratified by clinical presentation and previous statin therapy during the follow-up period.

Results: Fourteen trials with 3368 individuals were included in our meta-analysis. High-dose RSV preloading before $\mathrm{PCl}$ lead to a $58 \%$ reduction in MACE (odds ratio $[\mathrm{OR}]=0.42,95 \%$ confidence intervals [Cl]: 0.29-0.61, $P<0.00001$ ) and a $60 \%$ reduction in $\mathrm{PMI}(\mathrm{OR}=0.40,95 \% \mathrm{Cl}: 0.25-0.63, P<0.0001)$. This procedure also improved the final TIMI flow grade in patients undergoing $\mathrm{PCl}(\mathrm{OR}=1.61,95 \% \mathrm{Cl}: 1.09-2.38, P=0.02)$. The benefits on MACE were significant for both stable angina patients $(\mathrm{OR}=0.42,95 \% \mathrm{Cl}: 0.21-0.87, P=0.02)$ and acute coronary syndrome (ACS) patients $(\mathrm{OR}=0.42,95 \% \mathrm{Cl}: 0.27-0.65, P<0.0001)$; and for both statin naïve patients ( $\mathrm{OR}=0.42,95 \% \mathrm{Cl}: 0.28-0.64, P<0.0001)$ and previous statin therapy patients $(\mathrm{OR}=0.28,95 \% \mathrm{Cl}: 0.10-0.73, P=0.01)$.

Conclusion: High-dose RSV preloading can significantly improve myocardial perfusion and reduce both MACE and PMI in patients undergoing PCI. The cardioprotective benefits of RSV preloading were significant in not only stable angina and ACS patients but also statin naïve and previous statin therapy patients. The cardioprotective benefits of RSV preloading in the follow-up period mainly resulted from a reduction in spontaneous MI and TVR, especially for ACS and statin naïve patients.
\end{abstract}

Keywords: Rosuvastatin, Clinical events, Peri-procedural myocardial infarction, Meta-analysis

\footnotetext{
* Correspondence: lixd1894025@163.com

'Department of Cardiology, Shengjing Hospital of China Medical University,

Shenyang, China

Full list of author information is available at the end of the article
}

C Biomed Central

(c) 2015 Pan et al. Open Access This article is distributed under the terms of the Creative Commons Attribution 4.0 International License (http://creativecommons.org/licenses/by/4.0/), which permits unrestricted use, distribution, and reproduction in any medium, provided you give appropriate credit to the original author(s) and the source, provide a link to the Creative Commons license, and indicate if changes were made. The Creative Commons Public Domain Dedication waiver (http://creativecommons.org/publicdomain/zero/1.0/) applies to the data made available in this article, unless otherwise stated. 


\section{Introduction}

Percutaneous coronary intervention (PCI) is extensively used as a reperfusion strategy for coronary artery disease. Although this procedure is relatively safe and the procedure-related complications have markedly decreased with years, peri-procedural myocardial injury (PMI) can still occur [1]. The most common mechanism is side-branch occlusion during PCI, and distal embolism, coronary dissection, and inflammation can also result in PMI. Moreover, PMI lead to an increased incidence of death at follow-up (hazard ratio[HR] $=1.2$, $95 \%$ CI: 1.04-1.39) after adjustment for baseline covariates [2]. Research previously focused on the improvement of antithrombotic agents and vasodilators in decreasing the incidence of cardiac ischemic events during PCI, while recently it was found that pretreatment with statins may significantly reduce major adverse cardiac events (MACE) and PMI in patients undergoing PCI [3, 4]. It has already been proved in some metaanalyses of randomized controlled trials (RCTs) $[5,6]$. A meta-analysis of 3341 individuals from 13 randomized studies suggested that statin preloading leads to a significant decrease in 30-day MACE and PMI in patients undergoing PCI [5]. However, this research was not able to indicate whether statin pretreatment was effective in stable angina or acute coronary syndrome (ACS) patients, as patients were not assigned to subgroups according to their clinical presentation. Another updated meta-analysis comprising 5526 patients from 24 RCTs indicated that the cardioprotective benefits of statin preloading on MACE were effective for statin naïve or ACS patients [7]. However, recent published trials in which patients received high-dose rosuvastatin treatment prior to PCI were not included in this study, and this may have influenced the final clinical outcomes.

Rosuvastatin (RSV), an inhibitor of 3-hydroxy-3-methylglutaryl coenzyme A (HMG-CoA) reductase, has a number of pleiotropic effects, including antioxidative, antithrombotic, anti-inflammatory, and cardiovascular protective outcomes. Recently, investigators found a lower rate of MACE and PMI when patients undergoing PCI received high-dose RSV pretreatment [8-21]. However, most of the prospective trials lacked the power to detect differences from clinical outcomes, due to small size, varying endpoint definitions, individuals with different clinical presentation, and diverse regimens of RSV therapy. Therefore, we systematically evaluated the clinical benefits of high-dose RSV preloading prior to PCI by conducting a meta-analysis including all relevant RCTs.

\section{Methods}

\section{Search strategy}

In this study, we searched PubMed, EMBASE, the Cochrane Central Register of Controlled Trials and ISI
Web of Science databases up to June 2015 to determine prospective RCTs comparing the cardiovascular events of RSV preloading with control (placebo, no-statin, or current statin therapy) in patients undergoing PCI. Key words for searching were: "rosuvastatin", "percutaneous coronary intervention", "PCI", "stents", "angioplasty", "randomized" and "randomly". We also screened previous meta-analyses and the references of selected studies. No language restrictions were used.

\section{Study selection}

Trials were included if: (1) they were RCTs involving humans; (2) they selected patients with ACS (ST-segment elevation myocardial infarction [STEMI], unstable angina pectoris, or non-ST-segment elevation myocardial infarction [NSTEMI]) or stable angina; (3) they enrolled patients who were statin naïve or undergoing current therapy with statins; (4) they reported information on MACE including mortality, spontaneous myocardial infarction (MI), and target vessel revascularization (TVR) as well as PMI after PCI. Thrombolysis in myocardial infarction (TIMI) classification post-PCI was also recorded. Exclusion criteria included: (1) non-randomized controlled trials; (2) no outcomes of interest; (3) duplicate reports without additional or updated outcome data.

\section{Data extraction}

Two authors (YLP and YT) extracted the data in all the included trials, and any disagreement was resolved by discussion with the third author (BL). Firstly, the following information was extracted from each study: the first author's name, year of publication, sample size of the trial, type of population, clinical features, regimen of statins before and after PCI, study duration, and definition of PMI. Furthermore, we extracted the baseline characteristics and procedural details in all enrolled patients. Finally, we extracted data according to the clinical outcomes including mortality, spontaneous MI, TVR, and overall MACE during the follow-up period in each group.

\section{Quality assessment}

Study quality was evaluated based on the quality assessment criteria for RCTs in the Cochrane Handbook for Systematic Reviews of Interventions, including random sequence generation, allocation concealment, blinding of investigators, participants and outcome assessors, incomplete outcome data, selective reporting and other sources of bias.

\section{End-Points}

The primary end-points were MACE and PMI. We used the PMI definition which was taken from the original articles. Secondary end-point was final TIMI flow grade, 
which assessed myocardial perfusion after PCI. We carried out sub-group analyses according to the clinical condition (ACS and stable angina) or current statin therapy (statin naïve and previous statin therapy).

\section{Statistics}

Odds ratio (OR) with $95 \%$ confidence intervals (CI) was used to express dichotomous variables, such as the incidence of PMI, MACE, and post-PCI TIMI flow 3. Heterogeneity among studies was quantified using the $I^{2}$ statistic, defined as $I^{2}>50 \%$. In that case, the random effects model was used; otherwise, the fixed effect model was chosen. Funnel plots and Egger's regression test were used to illustrate the potential publication bias. Results were considered statistically significant at $P<0.05$. RevMan 5.3 software (Cochrane Collaboration, Copenhagen, Denmark) was used for statistical analysis.

\section{Results}

\section{Selected studies and baseline characteristics}

Based on the search strategy described above, 366 potentially relevant studies were initially included by titles and abstracts (Fig. 1), and 28 publications were selected. After reading the full texts of these publications, fourteen further studies were excluded as they were either duplicate studies, no outcome of interest reported, or were non-randomized studies. Finally, 3273 individuals from fourteen RCTs [8-21] were included, of which 1671 were randomized to the high-dose RSV group and 1602 were randomized to control.

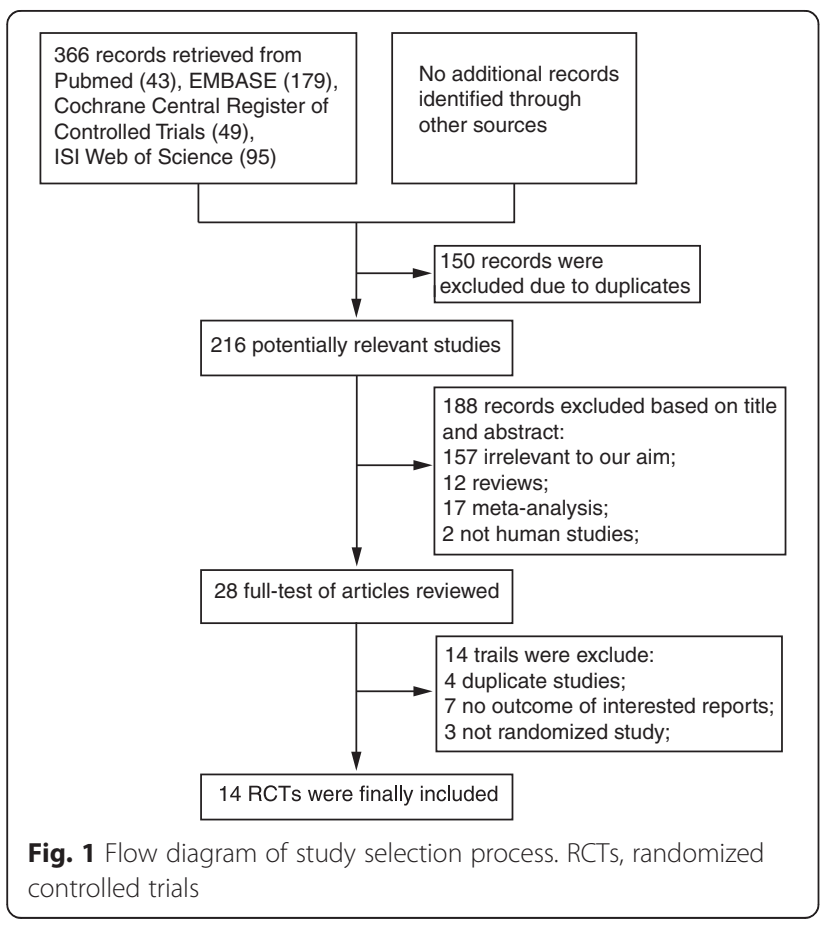

Table 1 shows the baseline characteristics of all the studies, which were published between 2010 and 2014 . There were ten trials $[8,10,11,13,15,17-21]$ with only statin naïve patients, one trial [16] with only previous statin therapy patients, and three trials $[9,12,14]$ with both of two. Five trials $[9,14-16,21]$ included stable angina patients, six trials $[8,11,13,17,19,20]$ included NSTE-ACS patients, two trials [12, 18] included STEMI patients, and one trial [10] included ACS patients. Thirteen trials $[8-13,15-21]$ included short-term RSV pretreatment (immediate to $24 \mathrm{~h}$ ), while one trial [14] included relatively long-term RSV pretreatment (5 to 7 days). Other characteristics of the included studies were follow-up duration and definition of PMI. The baseline characteristics of the patients and the procedural details in all trials are shown in Table 2. Approximately two-thirds of the overall population were male, three-fifths of those had hypertension, and one-third of those had diabetes. Preprocedural aspirin and clopidogrel were given to patients in all studies, and patients among four trials $[11,12,15,16]$ received a loading dose of clopidogrel $(600 \mathrm{mg})$, while the number of patients treated with clopidogrel in one trial [10] were unclear. Patients in a total of 11 trials [8,11-13,15-21] received glycoprotein IIb/IIIa inhibitors (GPIs) at the operators' discretion, while the remaining trials $[9,10,14]$ did not mention the use of GPIs. Almost four-fifths of the patients in seven trials $[8,10,13,15,17,20,21]$ underwent implantation of drug-eluting stents.

Judgments on each risk of bias item for all studies are shown in Fig. 2. Five of fourteen trials [10, 12, 15, 16, 19] reported the specific methods used for randomization, while the remaining included trials $[8,9,11,13,14,17,18$, $20,21]$ did not mention that. Three trials $[10,12,15]$ described the allocation concealments in detail, two trials $[11,14]$ did not use allocation concealments, and allocation concealments were not described in the remaining trials. $[8,9,13,16-21]$ Blinded methods were used in four trials, three of which $[10,12,13]$ used a blinded approach for investigators, participants and outcome assessors, while one trial [17] was only blinded for outcome assessors. None of the included studies had incomplete outcome data, selective reporting and other sources of bias.

\section{Effect of high-dose RSV preloading before $\mathrm{PCI}$ on coronary perfusion}

Effect of high-dose RSV preloading before PCI on post-PCI TIMI flow grade 3 were analyzed in ten trials $[9,10,13-15,17-21]$. Fixed effect model was chosen based on no potential heterogeneity across trials $\left(I^{2}=0 \%, P=0.91\right)$. The overall outcome indicated that RSV preloading lead to a $61 \%$ relative increase in post-PCI TIMI flow grade $3(\mathrm{OR}=1.61,95 \% \mathrm{CI}$ : 1.09-2.38, $P=0.02$; Fig. 3). 
Table 1 Characteristics of the included studies

\begin{tabular}{|c|c|c|c|c|c|c|c|}
\hline Study & $\begin{array}{l}\text { Patients } \\
\text { (RSV/ Con) }\end{array}$ & $\begin{array}{l}\text { Type of } \\
\text { population }\end{array}$ & $\begin{array}{l}\text { Clinical } \\
\text { feature }\end{array}$ & Timing before $\mathrm{PCl}$ & Regimen after $\mathrm{PCl}$ & Follow-up & Definition of PMl \\
\hline Gao [19] & $59 / 58$ & Statin naïve & NSTE-ACS & $\begin{array}{l}\text { RSV } 20 \mathrm{mg} 12 \mathrm{~h} \text { and } 10 \mathrm{mg} \\
2 \mathrm{~h} \text { before PCI VS placebo } \\
\text { treatment }\end{array}$ & $\begin{array}{l}\text { RSV } 10 \mathrm{mg} / \mathrm{d} \text { for } \\
\text { at least } 1 \text { year }\end{array}$ & 6 months & $C K M B>3 U N L$ \\
\hline Li [18] & $103 / 100$ & Statin naïve & STEMI & $\begin{array}{l}\text { RSV } 20 \mathrm{mg} \text { before } \mathrm{PCl} \\
\text { VS RSV } 10 \mathrm{mg} \text { treatment }\end{array}$ & $\begin{array}{l}\text { RSV } 10 \mathrm{mg} / \mathrm{d} \text { for } \\
3 \text { months }\end{array}$ & 30 days & $\mathrm{CKMB}>3 \mathrm{UNL}$ \\
\hline Luo [17] & $31 / 36$ & Statin naïve & NSTE-ACS & $\begin{array}{l}\text { RSV } 20 \mathrm{mg} 12 \mathrm{~h} \text { and } 20 \mathrm{mg} \\
2 \mathrm{~h} \text { before PCI VS no statin } \\
\text { pretreatment }\end{array}$ & RSV $10 \mathrm{mg} / \mathrm{d}$ & 30 days & $c T n l>3 U N L$ \\
\hline Takano [14] & $104 / 106$ & Mixed & $\begin{array}{l}\text { Stable } \\
\text { Angina }\end{array}$ & $\begin{array}{l}\text { RSV } 20 \text { mg 5-7day before } \\
\text { PCI VS RSV } 2.5 \text { mg treatment }\end{array}$ & $\begin{array}{l}\text { RSV } 10 \mathrm{mg} / \mathrm{d} \text { VS } \\
\text { RSV } 2.5 \mathrm{mg} / \mathrm{d}\end{array}$ & 12 months & $C K M B>3 U N L$ \\
\hline Wang [13] & $62 / 63$ & Statin naïve & NSTE-ACS & $\begin{array}{l}\text { RSV } 20 \mathrm{mg} \text { before } \mathrm{PCl} \\
\text { VS placebo pretreatment }\end{array}$ & $\begin{array}{l}\text { RSV } 10 \mathrm{mg} / \mathrm{d} \text { for } \\
\text { at least } 30 \text { days }\end{array}$ & 30 days & $\mathrm{CKMB}>3 \mathrm{UNL}$ \\
\hline Xie [8] & $79 / 80$ & Statin naïve & NSTE-ACS & $\begin{array}{l}\text { RSV } 20 \mathrm{mg} 12 \mathrm{~h} \text { and } 20 \mathrm{mg} \\
2 \mathrm{~h} \text { before PCI VS placebo } \\
\text { treatment }\end{array}$ & RSV $10 \mathrm{mg} / \mathrm{d}$ & 30 days & $c T n l>5 U N L$ \\
\hline Yun [20] & $225 / 220$ & Statin naïve & NSTE-ACS & $\begin{array}{l}\text { RSV } 40 \mathrm{mg} \text { before } \mathrm{PCl} \\
\text { VS no statin pretreament }\end{array}$ & RSV $10 \mathrm{mg} / \mathrm{d}$ & 12 months & $\mathrm{CKMB}>2 \mathrm{UNL}$ \\
\hline Veselka [9] & $220 / 225$ & Mixed & $\begin{array}{l}\text { Stable } \\
\text { Angina }\end{array}$ & $\begin{array}{l}\text { RSV } 20 \mathrm{mg} 12 \mathrm{~h} \text { and } 20 \mathrm{mg} \\
\text { before PCI VS no statin } \\
\text { pretreatment }\end{array}$ & Statin treatment & In hospital & $c T n l>5 U N L$ \\
\hline Cay [21] & $153 / 146$ & Statin naïve & $\begin{array}{l}\text { Stable } \\
\text { Angina }\end{array}$ & $\begin{array}{l}\text { RSV } 40 \text { mg } 24 \text { h before } \\
\text { PCI VS no RSV pretreatment }\end{array}$ & RSV $10-40 \mathrm{mg} / \mathrm{d}$ & In hospital & $C K M B>3 U N L$ \\
\hline Leoncini [11] & $252 / 252$ & Statin naïve & NSTE-ACS & $\begin{array}{l}\text { RSV } 40 \mathrm{mg} 24 \mathrm{~h} \text { and } 20 \mathrm{mg} \\
\text { before PCI VS no statin } \\
\text { pretreatment }\end{array}$ & $\begin{array}{l}\text { RSV } 20 \mathrm{mg} / \mathrm{d} \text { VS } \\
\text { atorvastatin } 40 \mathrm{mg} / \mathrm{d}\end{array}$ & 6 months & $\mathrm{CKMB}>3 \mathrm{UNL}$ \\
\hline ROMA [16] & $80 / 80$ & Statin naïve & $\begin{array}{l}\text { Stable } \\
\text { Angina }\end{array}$ & $\begin{array}{l}\text { RSV } 40 \mathrm{mg} 24 \mathrm{~h} \text { before } \mathrm{PCl} \\
\text { VS no RSV pretreatment }\end{array}$ & RSV $20 \mathrm{mg} / \mathrm{d}$ & 12 months & CKMB $>3$ UUNL \\
\hline ROMAll [15] & $175 / 100$ & $\begin{array}{l}\text { prior statin } \\
\text { treatment }\end{array}$ & $\begin{array}{l}\text { Stable } \\
\text { Angina }\end{array}$ & $\begin{array}{l}\text { RSV } 40 \mathrm{mg} 24 \mathrm{~h} \text { before } \mathrm{PCl} \\
\text { VS no statin pretreatment }\end{array}$ & RSV $20 \mathrm{mg} / \mathrm{d}$ & 12 months & $\mathrm{CKMB}>3 \mathrm{UNL}$ \\
\hline Ko [12] & $62 / 70$ & Mixed & STEMI & $\begin{array}{l}\text { RSV } 40 \text { mg before PCI VS } \\
\text { placebo treatment }\end{array}$ & $\begin{array}{l}\text { RSV } 40 \text { mg VS } 10 \mathrm{mg} \text { both for } \\
7 \text { days, and a further } 10 \mathrm{mg} / \mathrm{d} \\
\text { in both groups for } 3 \text { weeks }\end{array}$ & 3 months & NA \\
\hline Liang [10] & $66 / 66$ & Statin naïve & ACS & $\begin{array}{l}\text { RSV } 40 \text { mg } 4 \text { h VS RSV } 10 \\
\text { mg before PCl }\end{array}$ & $\begin{array}{l}\text { RSV } 10 \mathrm{mg} / \mathrm{d} \text { for at least } \\
1 \text { year }\end{array}$ & 6 months & NA \\
\hline
\end{tabular}

RSV rosuvastatin, $C K-M B$ creatine kinase-myocardial band, $C T n l$ cardiac troponin I, UNL upper normal limit, Mixed Statin naïve and prior statin treatment, PMI periprocedural myocardial infarction, ACS acute coronary syndrome, NSTE-ACS non-ST segment elevation ACS, STEMI ST segment elevation myocardial infarction, NA not available

\section{Effect of high-dose RSV preloading before $\mathrm{PCI}$ on clinical outcomes}

Effect of high-dose RSV preloading before PCI on MACE were analyzed in 12 trials [8, 10-20]. Fixed effect model was chosen based on no potential heterogeneity across trials $\left(I^{2}=0 \%, P=0.89\right)$. The overall outcome for MACE showed that RSV preloading lead to a $58 \%$ relative reduction in MACE $(\mathrm{OR}=0.42,95 \% \mathrm{CI}$ : 0.29-0.61, $P<0.00001$; Fig. 4). Effect of RSV preloading before PCI on PMI were analyzed in 12 trials [8, 9, 11, 13-21]. Random effects model was used due to substantial heterogeneity between the two groups $\left(I^{2}=53 \%, P=0.01\right)$. The overall outcome for PMI showed that RSV preloading lead to a $60 \%$ relative reduction in PMI $(\mathrm{OR}=0.40$, 95 \% CI: 0.25-0.63, $P<0.0001$; Fig. 5).

\section{Subgroup analyses with different types of coronary syndrome}

In order to assess the effect of high-dose RSV preloading in patients with different disease status before undergoing PCI, we divided all enrolled patients into two groups: stable angina and ACS. Due to the fixed effect model, RSV preloading before PCI lead to a $58 \%$ relative reduction in MACE for stable angina patients $(\mathrm{OR}=0.42$, $95 \%$ CI: $\left.0.21-0.87, P=0.02 ; I^{2}=10 \%\right)$; and a $58 \%$ relative reduction in MACE for ACS patients $(\mathrm{OR}=0.42$, 95 \% CI: $0.27-0.65, P<0.0001 ; I^{2}=0 \%$ ) (Fig. 4). We also evaluated the stable angina and ACS subgroup for their effects on PMI. Due to the random effects model, RSV preloading before PCI lead to a $63 \%$ reduction in PMI for stable angina patients $(\mathrm{OR}=0.37,95 \% \mathrm{CI}$ : 0.14-0.97, 
Table 2 Baseline clinical characteristics and procedural details in the overall population

\begin{tabular}{|c|c|c|}
\hline Variables & High-dose of RSV n/population (\%) & Control n/population (\%) \\
\hline Number of patients & 1671/3273 (51.1\%) & 1602/3273 (48.9\%) \\
\hline Male & 1150/1671 (68.8 \%) & 1075/1602 (67.1\%) \\
\hline Hypertension & 1088/1671 (65.1 \%) & 988/1602 (61.7\%) \\
\hline Diabetes Mellitus & $473 / 1671(28.3 \%)$ & 449/1602 (28.0 \%) \\
\hline Smoker & $592 / 1606(36.9 \%)$ & $573 / 1542(37.2 \%)$ \\
\hline Previous Ml & 223/1067 (20.9\%) & $224 / 1069(21.0 \%)$ \\
\hline Previous $\mathrm{PCl}$ & 160/1019 (15.7\%) & 143/1017 (14.1\%) \\
\hline Stable angina & 732/1671 (43.8\%) & $657 / 1602(41.0 \%)$ \\
\hline NSTEMI & 759/1671 (45.4\%) & 759/1602 (47.3\%) \\
\hline STEMI & 181/1671 (10.8\%) & 188/1602 (11.7\%) \\
\hline Multi-vessel disease & 198/644 (30.7\%) & 157/633 (24.8 \%) \\
\hline B2/C lesions & $654 / 918(71.2 \%)$ & $590 / 855(69.0 \%)$ \\
\hline Thrombus & 122/757 (16.1\%) & 135/763 (17.7 \%) \\
\hline LM & 16/700 (2.3 \%) & 25/709 (3.5\%) \\
\hline$\angle A D$ & $666 / 1253(53.2 \%)$ & $619 / 1174(52.7 \%)$ \\
\hline LCX & $360 / 1315(27.3 \%)$ & $354 / 1244(28.4 \%)$ \\
\hline RCA & 393/1253 (31.4\%) & $368 / 1174(31.3 \%)$ \\
\hline Multiple vessel PCl & 216/644 (33.5 \%) & 203/768 (26.4 \%) \\
\hline Aspirin & 1624/1671 (97.2 \%) & 1548/1602 (96.6 \%) \\
\hline Clopidogrel & 1599/1605 (99.6 \%) & 1530/1536 (99.6 \%) \\
\hline$\beta$-blockers & 952/1393 (68.3\%) & 979/1402 (69.8 \%) \\
\hline ACEI/ARB & 910/1393 (65.3\%) & 953/1402 (67.9\%) \\
\hline Glycoprotein IIb/IIla inhibitors & 76/1025 (7.4 \%) & 103/959 (10.7 \%) \\
\hline Numbers of DES & $560 / 696(80.5 \%)$ & $556 / 691(80.5 \%)$ \\
\hline
\end{tabular}

Previous MI previous myocardial infarction, Previous $P C I$ previous percutaneous coronary intervention, NSTE-ACS non-ST segment elevation acute coronary syndrome, STEMI ST segment elevation myocardial infarction, $L M$, left main, $L A D$ left anterior descending, $L C X$ left circumflex, RCA right coronary artery, DES drug-eluting stent

$\left.P=0.04 ; I^{2}=81 \%\right)$; and a $57 \%$ reduction in PMI for ACS patients $(\mathrm{OR}=0.43$, $95 \% \mathrm{CI}$ : 0.29-0.65, $P<0.0001$; $I^{2}=0 \%$ ) (Fig. 5).

\section{Subgroup analyses based on previous statin therapy}

In order to confirm whether current statin therapy before high-dose RSV preloading prior to PCI affected the incidence of MACE and PMI, we evaluated subgroups of patients based on their statin therapy before PCI in 11 trials $[8,10,11,13-20]$. Due to the fixed effect model, RSV preloading lead to a $60 \%$ relative reduction of MACE in all the enrolled patients $(\mathrm{OR}=0.40,95 \% \mathrm{CI}$ : $\left.0.27-0.58, P<0.00001 ; I^{2}=0 \%\right)$, a $58 \%$ relative reduction in the statin naïve group $(\mathrm{OR}=0.42$, $95 \% \mathrm{CI}$ : 0.28 $0.64, P<0.0001 ; I^{2}=0 \%$ ) and a $72 \%$ relative reduction in the previous statin therapy group $(\mathrm{OR}=0.28,95 \% \mathrm{CI}$ : $0.10-0.73, P=0.01 ; I^{2}=0 \%$ ) (Fig. 6). Due to the fixed effect model, RSV preloading lead to a $67 \%$ relative reduction of PMI in all the enrolled patients $(\mathrm{OR}=0.33$, 95 \% CI: $\left.0.25-0.45, P<0.00001 ; I^{2}=0 \%\right)$, a $66 \%$ relative reduction in the statin naïve group $(\mathrm{OR}=0.34,95 \% \mathrm{CI}$ : $0.24-0.49, P<0.00001 ; I^{2}=0 \%$ ), and a $69 \%$ relative reduction in the previous statin therapy group $(\mathrm{OR}=0.31$, 95 \% CI: 0.17-0.55, $P<0.0001 ; I^{2}=39$ \%) (Fig. 7).

\section{Effects of high-dose RSV preloading on follow-up outcome}

Table 3 depicts overall MACE including mortality, spontaneous MI, and TVR during follow-up. As mentioned above, the overall MACE was significantly reduced in all the enrolled patients. When stratified by type of disease, high-dose RSV preloading before PCI failed to decrease overall mortality $(P=0.42)$, spontaneous $\mathrm{MI} \quad(P=0.34)$ and TVR $(P=0.05)$ in patients with stable angina, and also didn't reduce overall mortality in ACS patients $(P=0.09)$. However, RSV preloading before PCI significantly reduced the incidence of spontaneous $\mathrm{MI}(P=0.02)$ and TVR $(P=0.01)$ in ACS patients. According to previous statin therapy, high-dose RSV preloading had no influence on overall mortality $(P=0.3)$, spontaneous MI 
a

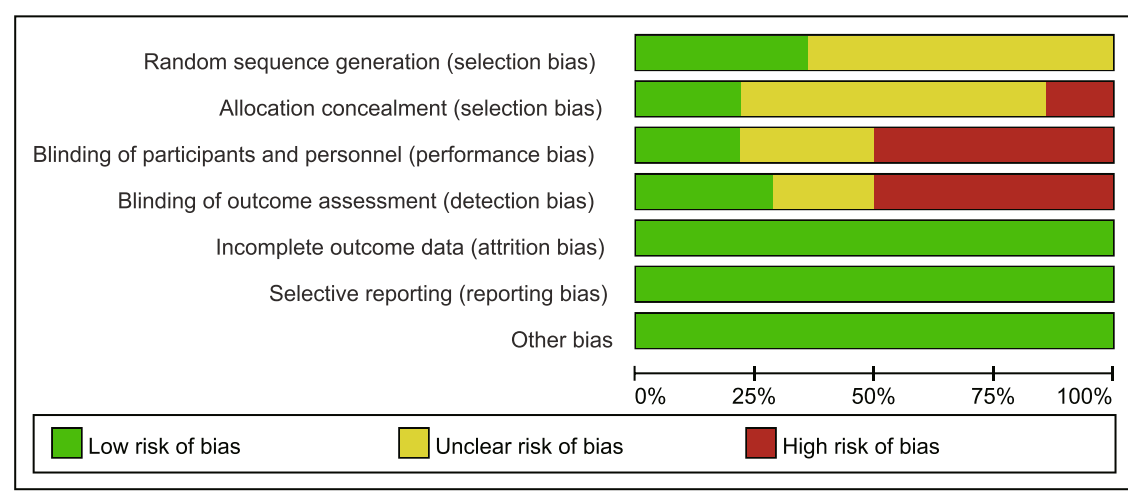

b

\begin{tabular}{|c|c|c|c|c|c|c|c|c|c|c|c|c|c|c|}
\hline $\begin{array}{l}\text { ડ } \\
\text { N } \\
\stackrel{0}{\Xi}\end{array}$ & $\begin{array}{l}\frac{x}{\overline{0}} \\
\stackrel{N}{\vec{A}} \\
\stackrel{\vec{A}}{ }\end{array}$ & $\begin{array}{l}\mathbf{s} \\
\stackrel{\Xi}{0} \\
\tilde{N} \\
\stackrel{\tilde{\omega}}{\omega}\end{array}$ & 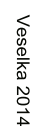 & 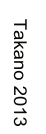 & 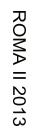 & 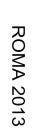 & $\begin{array}{l}\Sigma_{0} \\
\tilde{N} \\
\vec{\omega}\end{array}$ & 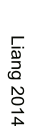 & $\begin{array}{l}\bar{r} \\
\hat{0} \\
\vec{\omega}\end{array}$ & 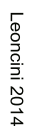 & 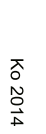 & $\begin{array}{l}0 \\
\stackrel{D}{0} \\
\tilde{N} \\
\stackrel{\vec{N}}{N}\end{array}$ & 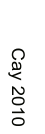 & \\
\hline$\sim$ & $\odot$ & $\sim$ & $\sim$ & $\odot$ & + & + & $\odot$ & + & $\sim$ & $\sim$ & + & + & $\odot$ & Random sequence generation (selection bias) \\
\hline$\sim$ & $\odot$ & $\sim$ & $\sim$ & 0 & $\sim$ & + & $\odot$ & + & $\sim$ & (1) & + & $\sim$ & $\sim$ & Allocation concealment (selection bias) \\
\hline (1) & $\odot$ & + & $\sim$ & 0 & (1) & (1) & $\odot$ & + & $\sim$ & (1) & + & (1) & (1) & Blinding of participants and personnel (performance bias) \\
\hline (1) & $\sim$ & + & $\sim$ & (1) & (1) & (1) & + & + & $\omega$ & (1) & + & (1) & (1) & Blinding of outcome assessment (detection bias) \\
\hline+ & + & + & + & + & + & + & + & + & + & + & + & + & + & Incomplete outcome data (attrition bias) \\
\hline+ & + & + & + & + & + & + & + & + & + & + & + & + & + & Selective reporting (reporting bias) \\
\hline+ & + & + & + & + & + & + & + & + & + & + & + & + & + & Other bias \\
\hline
\end{tabular}

Fig. 2 Quality assessment of included studies in this review $\mathbf{a}$. Risk of bias graph; b. Risk of bias summary

$(P=0.13)$ and TVR $(P=0.09)$ in previous statin therapy patients. Moreover, the overall mortality $(P=0.09)$ was not significantly reduced in statin naïve patients, while the incidence of spontaneous MI $(P=0.02)$ and TVR $(P=0.008)$ was significantly decreased.

\section{Publication bias}

As shown in Fig. 8, the results didn't provide any evidence of potential publication bias based on funnel plots and Egger's regression test. Funnel plots for MACE were generated using a fixed effect model $(P=0.139>0.05)$ and for PMI were generated using a random effects model $(P=0.273>0.05)$.

\section{Discussion}

Our analysis of 3273 individuals from 14 RCTs demonstated that high-dose RSV preloading before PCI can significantly improve the post-PCI TIMI flow grade and reduce the incidence of MACE and PMI. In addition, according to different disease presentations, high-dose RSV preloading can significantly decrease the incidence of MACE and PMI in both stable angina and ACS patients; when stratified according to current statin therapy, high-dose RSV preloading can also significantly reduce the incidence of MACE and PMI in both statin naïve and previous statin therapy patients. Moreover, according to the follow-up data, high-dose RSV preloading

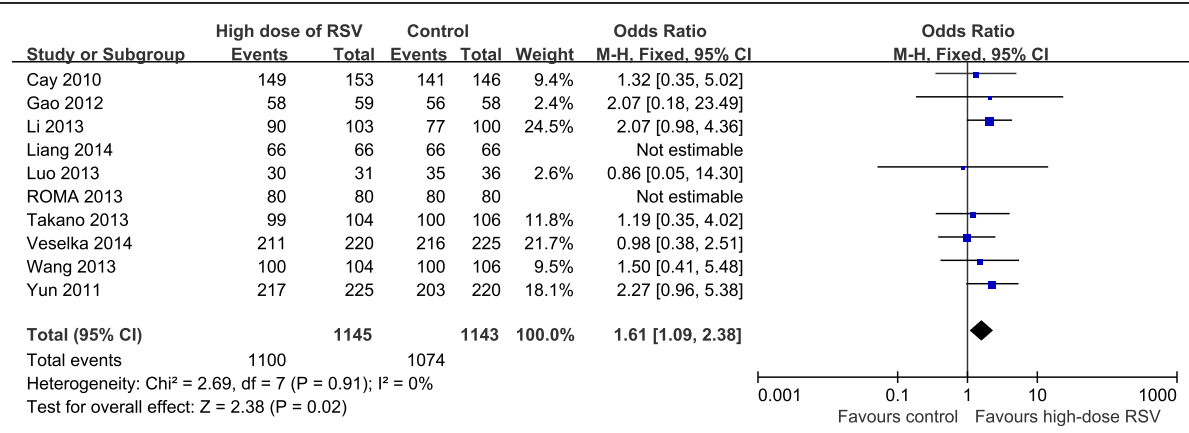

Fig. 3 ORs for final TIMI flow grade in overall population. Abbreviations: Cl, confidence interval; M-H, Mantel-Haenszel 


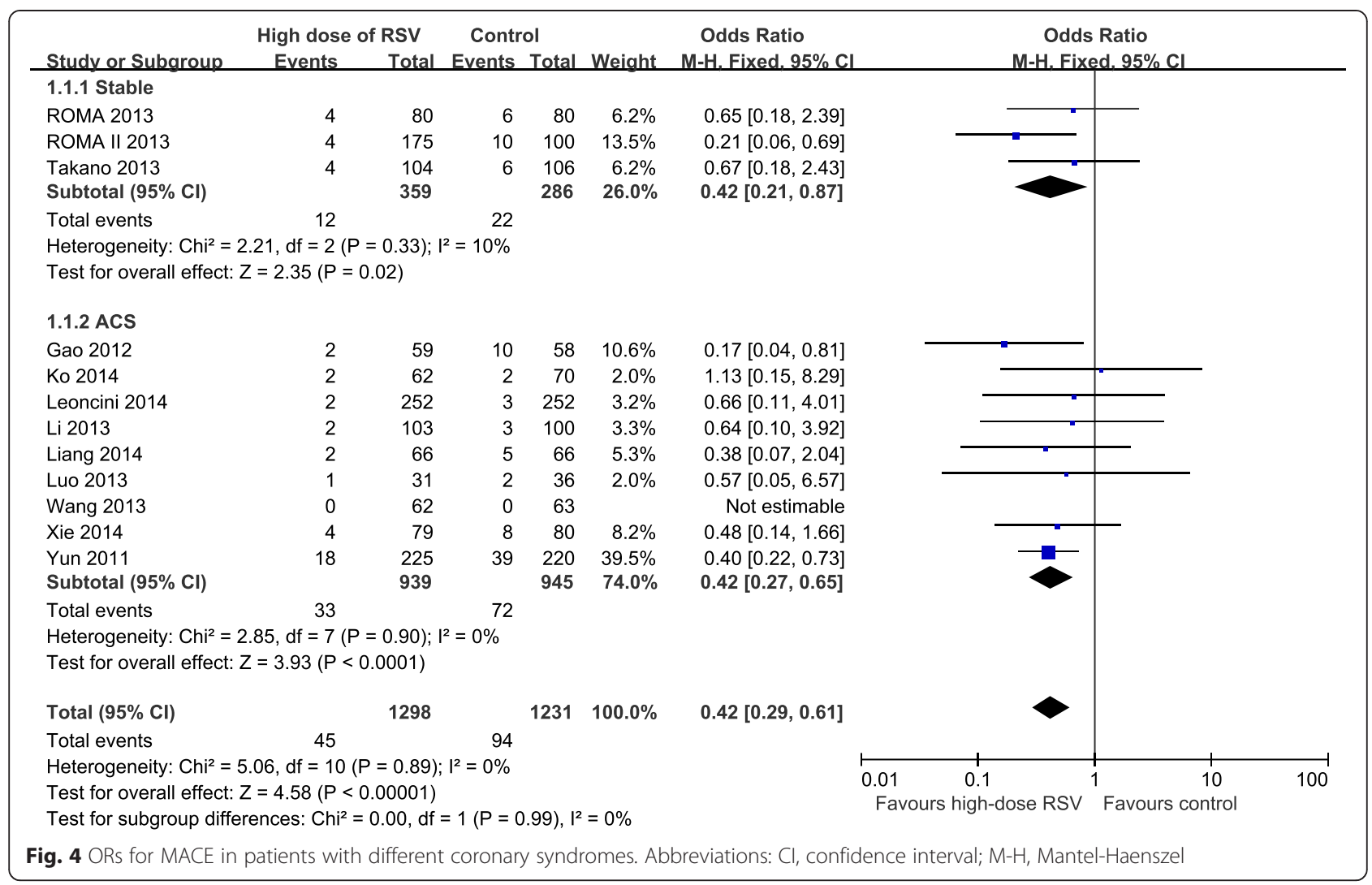

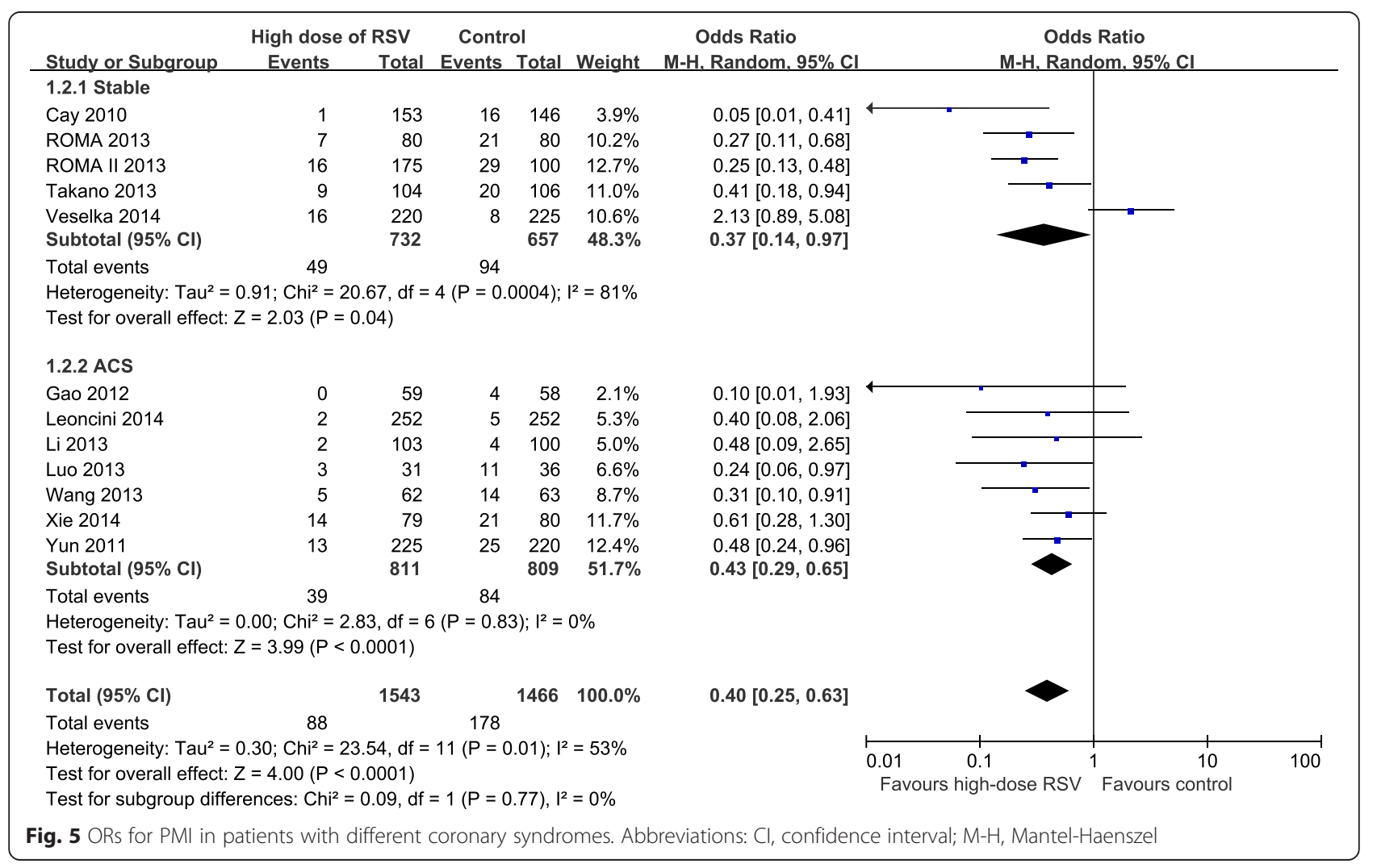




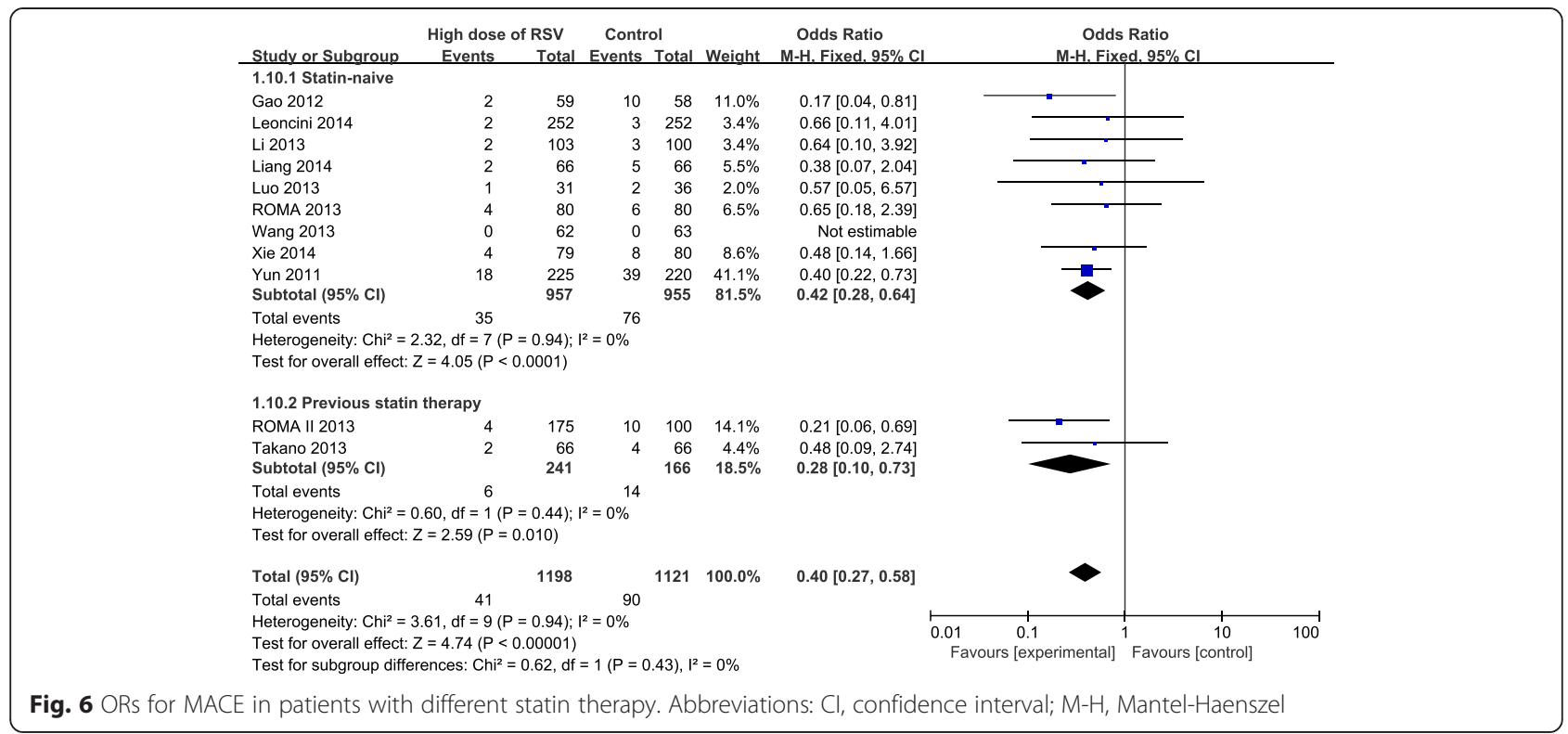

can significantly reduce the incidence of spontaneous MI and TVR in ACS and statin naïve patients, but failed to improve clinical outcomes in both patients with stable angina and current statin therapy.

We confirm and extend the meta-analyses by Benjo A.M [22] and Wang L [7] which indicated a reduction in PMI and MACE with statin preloading before PCI. Benjo et al. selected 1591 patients from 14 RCTs and found a $56 \%$ relative reduction in PMI and a $41 \%$ reduction in clinical events in patients with current statin treatment before PCI. Wang L et al. selected 24 RCTs with 5526 patients and demonstrated that statin preloading lead to a 59 \% relative reduction in PMI and a $39 \%$ relative reduction in MACE. These two studies were credible and conclusive, while different types of statins may have various effects on clinical events in both

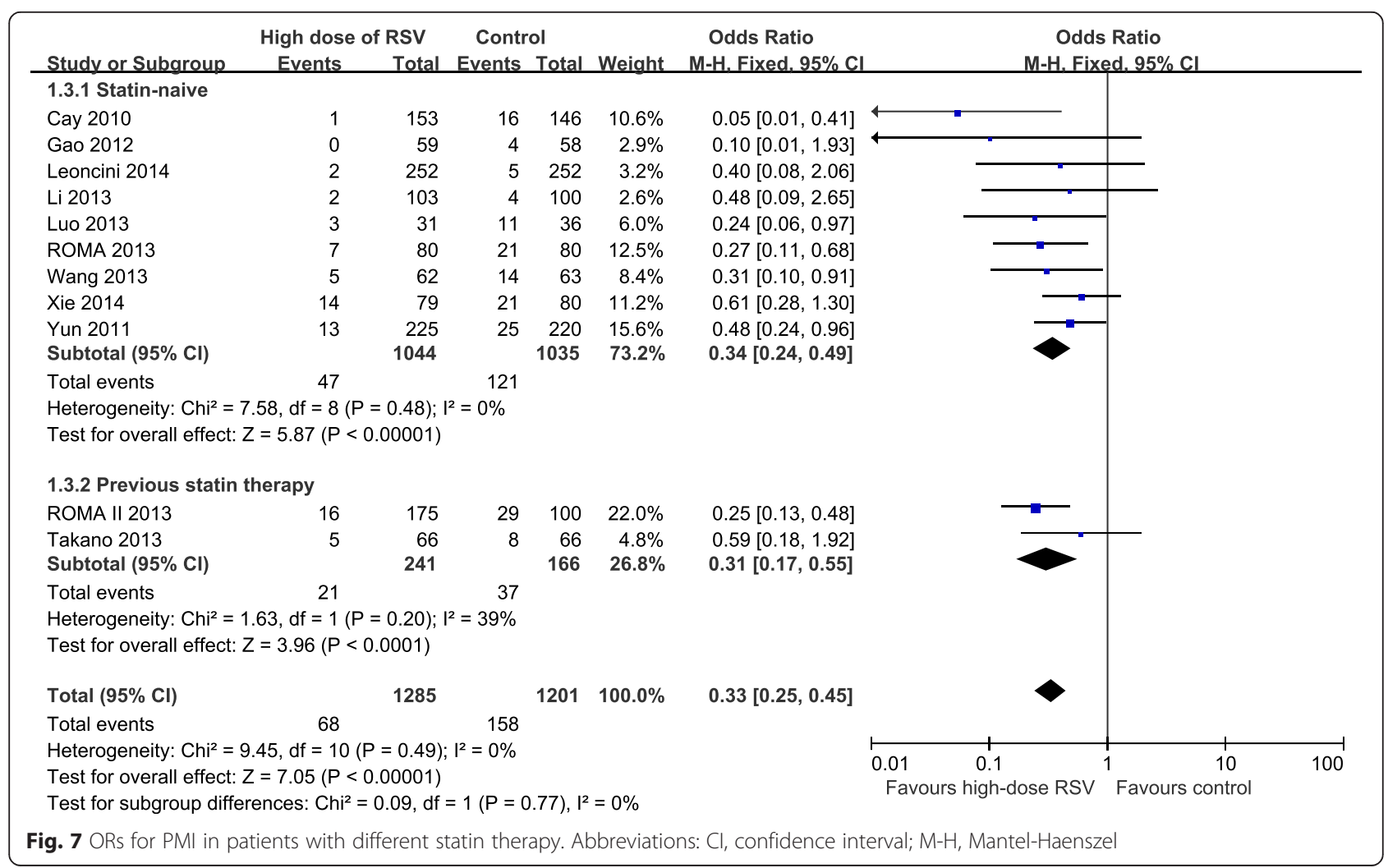


Table 3 Clinical events during follow-up

\begin{tabular}{|c|c|c|c|}
\hline Events & High-dose of RSV n (\%) & Control n (\%) & $P$ \\
\hline \multicolumn{4}{|l|}{ Stable } \\
\hline Death & $2(0.6 \%)$ & $3(1.0 \%)$ & 0.42 \\
\hline Spontaneous Ml & $5(1.4 \%)$ & $7(2.4 \%)$ & 0.34 \\
\hline TVR & $5(1.4 \%)$ & $12(4.2 \%)$ & 0.05 \\
\hline MACE & $12(3.3 \%)$ & $22(7.7 \%)$ & 0.02 \\
\hline \multicolumn{4}{|l|}{ ACS } \\
\hline Death & $7(0.7 \%)$ & $15(1.6 \%)$ & 0.09 \\
\hline Spontaneous Ml & $7(0.7 \%)$ & $20(2.1 \%)$ & 0.02 \\
\hline TVR & $19(2.0 \%)$ & $37(3.9 \%)$ & 0.01 \\
\hline MACE & $33(3.5 \%)$ & $72(7.6 \%)$ & $<0.0001$ \\
\hline \multicolumn{4}{|l|}{ Overall } \\
\hline Death & $9(0.7 \%)$ & $18(1.5 \%)$ & 0.06 \\
\hline Spontaneous Ml & $12(0.9 \%)$ & $27(2.2 \%)$ & 0.01 \\
\hline TVR & $24(1.8 \%)$ & $49(4.0 \%)$ & 0.002 \\
\hline MACE & $45(3.5 \%)$ & $94(7.6 \%)$ & $<0.00001$ \\
\hline \multicolumn{4}{|l|}{ Statin naïve } \\
\hline Death & $7(0.7 \%)$ & $15(1.6 \%)$ & 0.09 \\
\hline Spontaneous Ml & $8(0.8 \%)$ & $21(2.2 \%)$ & 0.02 \\
\hline TVR & $20(2.1 \%)$ & $40(4.2 \%)$ & 0.008 \\
\hline MACE & $35(3.7 \%)$ & $76(8.0 \%)$ & $<0.0001$ \\
\hline \multicolumn{4}{|l|}{ Prior statin treatment } \\
\hline Death & $1(0.4 \%)$ & $2(1.2 \%)$ & 0.3 \\
\hline Spontaneous MI & $2(0.8 \%)$ & $5(3.0 \%)$ & 0.13 \\
\hline TVR & $3(1.2 \%)$ & $7(4.2 \%)$ & 0.09 \\
\hline MACE & $6(2.4 \%)$ & $14(8.4 \%)$ & 0.01 \\
\hline \multicolumn{4}{|l|}{ Overall } \\
\hline Death & $8(0.7 \%)$ & $17(1.5 \%)$ & 0.05 \\
\hline Spontaneous MI & $10(0.8 \%)$ & $26(2.3 \%)$ & 0.005 \\
\hline TVR & 23(1.9\%) & $47(4.2 \%)$ & 0.002 \\
\hline MACE & $41(3.4 \%)$ & $90(8.0 \%)$ & $<0.00001$ \\
\hline
\end{tabular}

ACS acute coronary syndrome, Spontaneous $\mathrm{MI}$ spontaneous myocardial infarction, TVR target vessel revascularization, MACE major adverse cardiovascular events

studies. Although the former study was powerful and proved their hypothesis, patients with STEMI and current statin treatment were excluded, which led to the lack of identifying the effects of statin preloading in the overall population. Data on patients with chronic statin therapy were included in the latter study, however, recent trials on high-dose RSV pretreatment, which were published after this meta-analysis, drew controversial conclusions concerning the cardioprotective benefits of this treatment on PMI and MACE. Our meta-analysis demonstrated that RSV preloading before PCI lead to a $60 \%$ relative reduction in PMI $(P<0.0001)$ and a $58 \%$ relative reduction in MACE $(P<0.00001)$. The cardioprotective effects of RSV were consistent in not only stable angina and ACS patients but also statin naïve and previous statin therapy patients. High-dose RSV preloading can significantly reduce the clinical outcome in the overall population, which was mainly due to a reduction of TVR, especially for ACS and statin naïve patients. Although these benefits of RSV preloading failed to decrease overall mortality in the enrolled patients, they reduced the incidence of spontaneous MI and TVR in ACS and statin naïve patients.

PMI is characterized by any cardiac serum marker (creatine kinase-myocardial band [CK-MB]; cardiac troponin I [cTnI]) elevation three times or more the upper limit of normal (ULN) after PCI, which has been shown to occur in approximately $15-20 \%$ of patients undergoing PCI procedures in spite of different definitions used [23]. The pathogenic mechanisms of PMI include side-branch occlusion, distal embolism, endothelial dysfunction, oxidative stress, and inflammation [1]. In order to reduce the incidence of PMI, researchers have focused on antiplatelet agents, anticoagulants, vasodilators, and beta-blockers to improve coronary blood flow after PCI. More recently, some studies [21, 24, 25] have suggested that the reduction of the MACE and PMI in patients undergoing PCI was associated with HMG-CoA reductase inhibitors (statins), in particular atorvastatin and RSV. In addition to its lipid-lowering effects, RSV exerts many cardioprotective effects including an improvement in endothelial function, antithrombotic actions, inhibition of thrombosis, plaque stabilization, and suppression of inflammation. These may partly account for the cardioprotective effects of RSV on spontaneous MI and TVR in ACS patients due to the high inflammatory status in these patients, which is associated with a high predictive value for the occurrence of MACE [26].

The possible mechanisms underlying the early protective action of RSV are unclear, as the enrolled patients were given a short-term high-dose of RSV before PCI, which may not have had a significant influence on cholesterol level. Vilahur G et al. [27] showed that RSV enhanced PKC, Erk2, AKT/PKB signal pathways and its downstream effectors to attenuate inflammation and cardiomyocyte apoptosis in the peri-infarcted zone and reduce infarct size in pigs. RSV has been shown to enhance the protective effects of ischemic post-conditioning against myocardial ischemia and reperfusion (I/R) injury in rats via activating PI3K/Akt/eNOS signaling pathway [28]. Nitrous oxide (NO), due to the activation of endothelial nitric oxide synthase (eNOS), can decrease leukocyte activation and infiltration, platelet activation and aggregation, vasoconstriction and contractile dysfunction [1]. These beneficial cardiac effects of statins are absent in eNOS knockout mice and can be reversed by using the specific inhibitor of PI3K kinase and eNOS $[29,30]$. In our meta-analysis, cardiac benefits of high- 

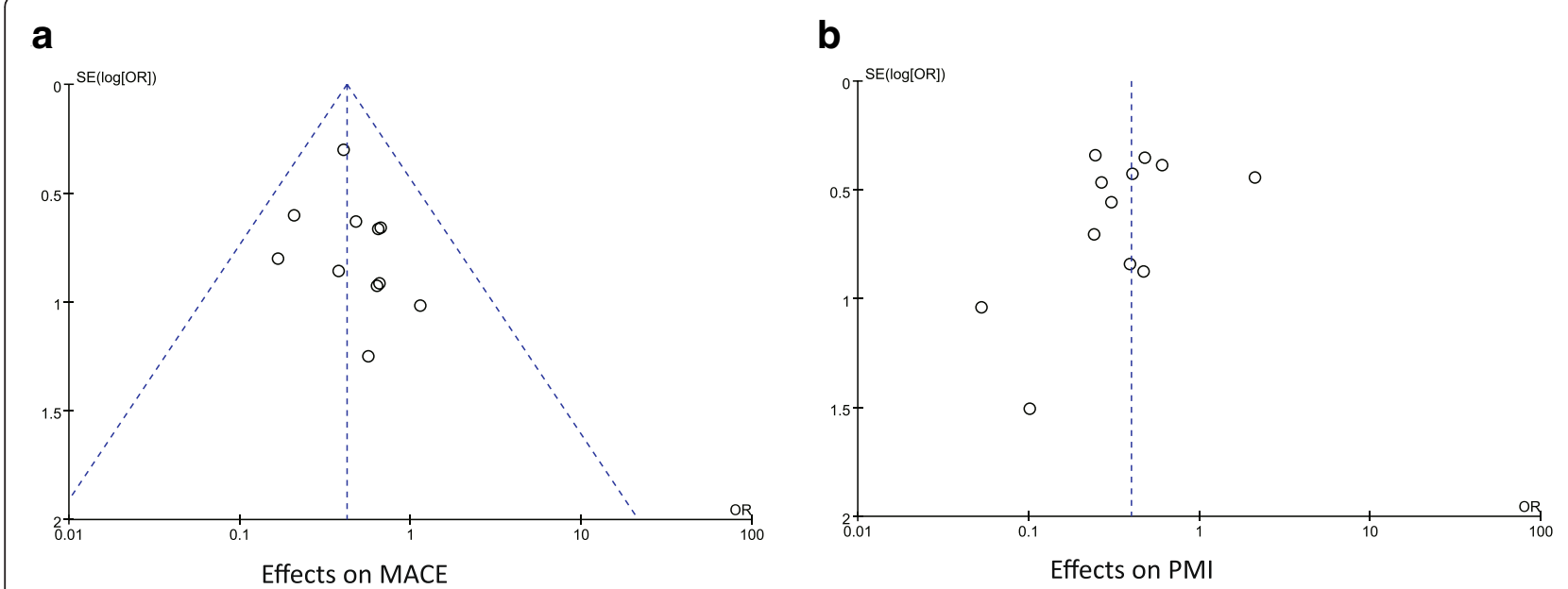

Fig. 8 Funnel plots of the included studies. a. Funnel plots for MACE; $\boldsymbol{b}$. Funnel plots for PMI. The results show no potential publication bias for MACE and PMI. Abbreviations: OR, odds ratio; SE, standard error

dose RSV preloading before PCI decreased the incidence of spontaneous MI and TVR in statin naïve patients, but had no effects on previous statin therapy patients. Patients with previous statin therapy are probably in a condition of dyslipidaemia. They are likely to take some nutraceuticals (resveratrol, grape seed, curcumin, zinc, and fish oil) and antioxidants (carotenoids, vitamins $\mathrm{A}, \mathrm{C}$, and $\mathrm{E}$ ) in their daily diet to decrease the plasma lipids, which can influence the overall outcomes [31, 32]. Hence, we speculate that the cardiovascular benefits of short-term high-dose RSV preloading might be counteracted by long-term intake of statin, nutraceuticals, or antioxidants. Interestingly, chronic statin therapy failed to exert a cardioprotective effect that wanes with time associated with increased levels of PTEN (phosphatase and tensin homolog deleted on chromosome ten, an inhibitor of PI3K) in SD rats and these can partly attenuate the cardioprotective effects of high-dose RSV preloading, which may partially confirm our speculation [33].

The results of our study are different from those by Veselka et al. [9], who demonstrated that high-dose RSV therapy had no effects on the incidence of PMI in patients with stable angina. We compared the results when included and excluded Veselka's trial data. The significant effects on PMI were unchanged, while excluding Veselka's data changed the homogeneity in the stable angina group $\left(I^{2}=81\right.$ to $\left.16 \%\right)$ and the overall population $\left(I^{2}=53\right.$ to $\left.0 \%\right)$. We indicated that the main heterogeneity was due to the different doses and types of statins administered. 36 of 220 patients (16.4\%) in the high-dose RSV group and 51 of 225 patients $(22.6 \%)$ in control received long-term high-dose statin therapy (atorvastatin 40 or $80 \mathrm{mg}$, RSV 20 or $40 \mathrm{mg}$ ). This may partially explain why high-dose RSV treatment before PCI failed to reduce the incidence of PMI in their study, as the beneficial cardioprotection effect of transient high-dose RSV may be eliminated by chronic high-dose statin therapy.

Statin therapy is only recommended as secondary prevention of cardiovascular outcomes in the present guidelines for ACS and PCI $[34,35]$. However, high-dose RSV preloading before $\mathrm{PCI}$ can reduce the incidence of MACE and PMI, which is not recommended in these guidelines. Our analysis adds strength and power to current recommendations and potentially expands the use of RSV before PCI.

\section{Study limitations}

There were several limitations in our meta-analysis. Firstly, the trials included did not use a uniform RSV regimen, definition of PMI, or clinical outcomes examined. Different doses and duration of RSV treatment in patients with different backgrounds may have various effects on MACE and PMI. Secondly, seven of fourteen trials included not long enough follow-up period which was no more than 30 days of observation. Hence, more high quality RCTs are required to identify the beneficial cardiac effects of RSV preloading before PCI over a longer follow-up period. Thirdly, due to a lack of patient-level data, we failed to analyze the effect of high-dose RSV preloading on peri-procedural high-sensitivity $\mathrm{C}$-reactive protein (hs-CRP) level variation (post-intervention hs-CRP minus baseline hs-CRP). In the same way, we are not able to provide subgroup analyses based on gender due to a lack of patient-level data. Hence, further studies are required to investigate whether high-dose RSV preloading plays a different role in men and women. Finally, there was potential heterogeneity in our study due to limited study numbers, small sample sizes, different protocols, and patients with various backgrounds. 


\section{Conclusion}

High-dose RSV preloading can significantly improve myocardial perfusion and reduce MACE and PMI in patients undergoing PCI. The cardioprotective effects of RSV preloading were significant in not only stable angina and ACS patients but also statin naïve and previous statin therapy patients. The cardioprotective effects of high-dose RSV were mainly due to the reduction in spontaneous MI and TVR, especially in ACS and statin naive patients. Therefore, it indicates that RSV preloading before PCI should be used in consideration of the disease presentation and current statin therapy in patients before undergoing PCI.

\begin{abstract}
Abbreviations
RSV: Rosuvastatin; PCl: Percutaneous coronary intervention; PMl: Peri-procedural myocardial injury; MACE: Major adverse cardiac events; RCTs: Randomized controlled trials; MI: Myocardial infarction; TVR: Target vessel revascularization; HR: Hazard ratio; OR: Odds ratio; Cl: Confidence intervals; ACS: Acute coronary syndrome; HMG-COA: 3-hydroxy-3-methylglutaryl coenzyme A; TIMl: Thrombolysis in myocardial infarction; hs-CRP: High-sensitivity C-reactive protein; GPIs: Glycoprotein IIb/Illa inhibitors; ULN: Upper limit of normal; I/R injury: Ischemia and reperfusion injury; NO: Nitrous oxide; eNOS: Nitric oxide synthase; PTEN: Phosphatase and tensin homolog deleted on chromosome ten; CK-MB: Creatine kinase-myocardial band; CTnl: cardiac troponin I; NSTE-ACS: Non-ST segment elevation ACS; STEMI: ST segment elevation myocardial infarction; NA: Not available; LM: Left main; LAD: Left anterior descending; LCX: Left circumflex; RCA: Right coronary artery; DES: Drug-eluting stent; $\mathrm{M}-\mathrm{H}$ : Mantel-Haenszel; SE: Standard error.
\end{abstract}

\section{Competing interests}

The authors declare that they have no competing interests.

\section{Authors' contributions}

XDL contributed to the overall design of the study. YLP and YT performed the data analysis, interpreted the results. YLP wrote section of the initial draft. $\mathrm{BL}$ helped with the data analysis. All authors read and approved the final version of the manuscript.

\section{Acknowledgments}

This study was funded by the Funding Program of Liaoning Educational Committee (Grant No. L2013314).

\section{Author details}

'Department of Cardiology, Shengjing Hospital of China Medical University, Shenyang, China. ${ }^{2}$ Department of Anesthsia, Shengjing Hospital of China Medical University, Shenyang, China.

\section{Received: 5 August 2015 Accepted: 16 August 2015}

Published online: 27 August 2015

\section{References}

1. Herrmann J. Peri-procedural myocardial injury: 2005 update. Eur Heart J. 2005:26:2493-519.

2. Erbel R, Wijns W. The Year in Cardiology 2013: coronary intervention. Eur Heart J. 2014;35:313-20.

3. Tentzeris I, Rohla M, Jarai R, Farhan S, Freynhofer MK, Unger G, et al. Influence of High-Dose Highly Efficient Statins on Short-Term Mortality in Patients Undergoing Percutaneous Coronary Intervention With Stenting for Acute Coronary Syndromes. Am J Cardiol. 2014;113:1099-104.

4. Kim JW, Yun KH, Kim EK, Kim YC, Joe D-Y, Ko JS, et al. Effect of High Dose Rosuvastatin Loading before Primary Percutaneous Coronary Intervention on Infarct Size in Patients with ST-Segment Elevation Myocardial Infarction. Korean Circulation J. 2014:44:76-81.

5. Patti G, Cannon CP, Murphy SA, Mega S, Pasceri V, Briguori C, et al. Clinical benefit of statin pretreatment in patients undergoing percutaneous coronary intervention: A collaborative patient-level meta-analysis of 13 randomized studies. Circulation. 2011;123:1622-32.

6. Winchester DE, Wen X, Xie L, Bavry AA. Evidence of pre-procedural statin therapy: A meta-analysis of randomized trials. J Am Coll Cardiol. 2010;56:1099-109.

7. Wang L, Peng P, Zhang O, Xu X, Yang S, Zhao Y, et al. High-dose statin pretreatment decreases periprocedural myocardial infarction and cardiovascular events in patients undergoing elective percutaneous coronary intervention: A meta-analysis of twenty-four randomized controlled trials. PLoS ONE. 2014;9, e113352.

8. Xie W, Li P, Wang Z, Chen J, Lin Z, Liang X, et al. Rosuvastatin may reduce the incidence of cardiovascular events in patients with acute coronary syndromes receiving percutaneous coronary intervention by suppressing miR-155/SHIP-1 signaling pathway. Cardiovasc Ther. 2014;32:276-82.

9. Veselka J, Hajek P, Tomasov P, Tesar D, Bruhova H, Matejovic M, et al. Effect of rosuvastatin therapy on troponin I release following percutaneous coronary intervention in nonemergency patients (from the TIP 3 study). Am J Cardiol. 2014;113:446-51.

10. Liang D, Zhang Q, Yang H, Zhang R, Yan W, Gao H, et al. Anti-oxidative stress effect of loading-dose rosuvastatin prior to percutaneous coronary intervention in patients with acute coronary syndrome: a prospective randomized controlled clinical trial. Clin Drug Investig. 2014;34:773-81.

11. Leoncini M, Toso A, Maioli M, Tropeano F, Villani S, Bellandi F. Early high-dose rosuvastatin for contrast-induced nephropathy prevention in acute coronary syndrome: Results from the PRATO-ACS Study (Protective Effect of Rosuvastatin and Antiplatelet Therapy On contrast-induced acute kidney injury and myocardial damage in patients with Acute Coronary Syndrome). J Am Coll Cardiol. 2014;63:71-9.

12. Ko Y-G, Won H, Shin D-H, Kim J-S, Kim B-K, Choi D, et al. Efficacy of Early Intensive Rosuvastatin Therapy in Patients With ST-Segment Elevation Myocardial Infarction Undergoing Primary Percutaneous Coronary Intervention (ROSEMARY Study). Am J Cardiol. 2014;114:29-35.

13. Wang Z, Dai $H$, Xing M, Yu Z, Lin X, Wang $S$, et al. Effect of a single high loading dose of rosuvastatin on percutaneous coronary intervention for acute coronary syndromes. J Cardiovasc Pharmacol Ther. 2013;18:327-33.

14. Takano H, Ohba T, Yamamoto E, Miyachi H, Inui K, Kawanaka H, et al. Usefulness of rosuvastatin to prevent periprocedural myocardial injury in patients undergoing elective coronary intervention. Am J Cardiol. 2013;111:1688-93.

15. Sardella G, Lucisano L, Mancone M, Conti G, Calcagno S, Stio RE, et al. Comparison of high reloading ROsuvastatin and Atorvastatin pretreatment in patients undergoing elective $\mathrm{PCI}$ to reduce the incidence of MyocArdial periprocedural necrosis. The ROMA II trial. Int J Cardiol. 2013;168:3715-20.

16. Sardella G, Conti G, Donahue M, Mancone M, Canali E, Carlo C, et al. Rosuvastatin pretreatment in patients undergoing elective $\mathrm{PCI}$ to reduce the incidence of myocardial periprocedural necrosis: The ROMA trial. Catheter Cardiovasc Interv. 2013;81:E36-43.

17. Luo J, Li J, Shen X, Hu X, Fang Z, Lv X, et al. The effects and mechanisms of high loading dose rosuvastatin therapy before percutaneous coronary intervention in patients with acute coronary syndrome. Int J Cardiol. 2013;168:2350-3.

18. Li P, Huang C, Gan J, Liang X, Chen J, Wang Z. Efficacy of high-dose rosuvastatin loading before primary percutaneous coronary intervention in ST-segment elevation myocardial infarction. Chin J Clin Pharmacol. 2013;29:732-5.

19. Gao Y, Jia Z-m, Sun Y-j, Zhang Z-h, Ren L-n, Qi G-x. Effect of high-dose rosuvastatin loading before percutaneous coronary intervention in female patients with non-ST-segment elevation acute coronary syndrome. Chin Med J. 2012;125:2250-4.

20. Yun KH, Oh SK, Rhee SJ, Yoo NJ, Kim NH, Jeong JW. 12-month follow-up results of high dose rosuvastatin loading before percutaneous coronary intervention in patients with acute coronary syndrome. Int J Cardiol. 2011;146:68-72.

21. Cay S, Cagirci G, Sen N, Balbay Y, Durmaz T, Aydogdu S. Prevention of peri-procedural myocardial injury using a single high loading dose of rosuvastatin. Cardiovasc Drugs Ther. 2010;24:41-7.

22. Benjo AM, El-Hayek GE, Messerli F, DiNicolantonio JJ, Hong MK, Aziz EF, et al. High dose statin loading prior to percutaneous coronary intervention decreases cardiovascular events: A meta-analysis of randomized controlled trials. Catheter Cardiovasc Interv. 2015;85:53-60. 
23. Norris DM, Anderson JR. Statin loading before percutaneous coronary intervention to reduce periprocedural myocardial infarction. Cardiol Rev. 2012;20:319-24.

24. Di Sciascio G, Patti G, Pasceri V, Gaspardone A, Colonna G, Montinaro A. Efficacy of atorvastatin reload in patients on chronic statin therapy undergoing percutaneous coronary intervention: results of the ARMYDA-RECAPTURE (Atorvastatin for Reduction of Myocardial Damage During Angioplasty) Randomized Trial. J Am Coll Cardiol. 2009;54:558-65.

25. Patti G, Pasceri V, Colonna G, Miglionico M, Fischetti D, Sardella G, et al. Atorvastatin pretreatment improves outcomes in patients with acute coronary syndromes undergoing early percutaneous coronary intervention: results of the ARMYDA-ACS randomized trial. J Am Coll Cardiol. 2007:49:1272-8

26. Yun $\mathrm{KH}$, Jeong MH, Oh SK, Rhee SJ, Park EM, Lee EM, et al. Response of high-sensitivity C-reactive protein to percutaneous coronary intervention in patients with acute coronary syndrome. Heart Vessels. 2009;24:175-80.

27. Vilahur G, Casani L, Pena E, Duran X, Juan-Babot O, Badimon L. Induction of RISK by HMG-COA reductase inhibition affords cardioprotection after myocardial infarction. Atherosclerosis. 2009;206:95-101.

28. Cai W, Fang J, Chen Z, Lin Y, Wu L, Chen L. Rosuvastatin enhances the protective effects of ischemic postconditioning on myocardial ischaemia-reperfusion injury in type 2 diabetic rat. Chin J Cardiol. 2010;38:814-8

29. Bell RM, Yellon DM. Atorvastatin, administered at the onset of reperfusion, and independent of lipid lowering, protects the myocardium by up-regulating a pro-survival pathway. J Am Coll Cardiol. 2003;41:508-15.

30. Atar S, Ye Y, Lin Y, Freeberg SY, Nishi SP, Rosanio S, et al. Atorvastatin-induced cardioprotection is mediated by increasing inducible nitric oxide synthase and consequent S-nitrosylation of cyclooxygenase-2. Am J Physiol Heart Circ Physiol. 2006;290:H1960-8.

31. Ciccone MM, Cortese F, Gesualdo M, Carbonara S, Zito A, Ricci G, et al. Dietary Intake of Carotenoids and Their Antioxidant and Anti-Inflammatory Effects in Cardiovascular Care. Mediators Inflamm. 2013;2013:782137.

32. Scicchitano P, Cameli M, Maiello M, Modesti PA, Muiesan ML, Novo S, et al. Nutraceuticals and dyslipidaemia: Beyond the common therapeutics. J Funct Foods. 2014;6:11-32.

33. Mensah $\mathrm{K}$, Mocanu MM, Yellon DM. Failure to protect the myocardium against ischemia/reperfusion injury after chronic atorvastatin treatment is recaptured by acute atorvastatin treatment: a potential role for phosphatase and tensin homolog deleted on chromosome ten? J Am Coll Cardiol. 2005;45:1287-91.

34. Amsterdam EA, Wenger NK, Brindis RG, Casey Jr DE, Ganiats TG, Holmes Jr $D R$, et al. AHA/ACC guideline for the management of patients with non-ST-elevation acute coronary syndromes: a report of the American College of Cardiology/American Heart Association Task Force on Practice Guidelines. Circulation. 2014:2014(130):e344-426.

35. Kolh P, Windecker S, Alfonso F, Collet JP, Cremer J, Falk V, et al. ESC/EACTS Guidelines on myocardial revascularization: the Task Force on Myocardial Revascularization of the European Society of Cardiology (ESC) and the European Association for Cardio-Thoracic Surgery (EACTS). Developed with the special contribution of the European Association of Percutaneous Cardiovascular Interventions (EAPCI). Eur J Cardiothorac Surg. 2014:2014(46):517-92.

\section{Submit your next manuscript to BioMed Central and take full advantage of:}

- Convenient online submission

- Thorough peer review

- No space constraints or color figure charges

- Immediate publication on acceptance

- Inclusion in PubMed, CAS, Scopus and Google Scholar

- Research which is freely available for redistribution

Submit your manuscript at www.biomedcentral.com/submit 